\title{
La conservation-restauration du patrimoine technique et industriel dans le cadre de la loi sur les Musées de France, une mission impossible?
}

The conservation-restoration of the technical and industrial heritage within the framework of the law relating to French museums, an impossible mission?

\section{Agnès Mirambet-Paris et François Mirambet}

\section{OpenEdition}

Journals

Édition électronique

URL : http://journals.openedition.org/ocim/874

DOI : $10.4000 /$ ocim. 874

ISSN : 2108-646X

Éditeur

OCIM

Édition imprimée

Date de publication : 1 mai 2011

Pagination : 27-35

ISSN : 0994-1908

Référence électronique

Agnès Mirambet-Paris et François Mirambet, «La conservation-restauration du patrimoine technique et industriel dans le cadre de la loi sur les Musées de France, une mission impossible? », La Lettre de I'OCIM [En ligne], 135 | 2011, mis en ligne le 01 mai 2013, consulté le 19 avril 2019. URL : http:// journals.openedition.org/ocim/874; DOI : 10.4000/ocim.874 


\title{
La conservation-restauration du patrimoine technique et industriel dans le cadre de la loi sur les Musées de France, une mission impossible?
}

\author{
Agnès Mirambet-Paris et François Mirambet *
}

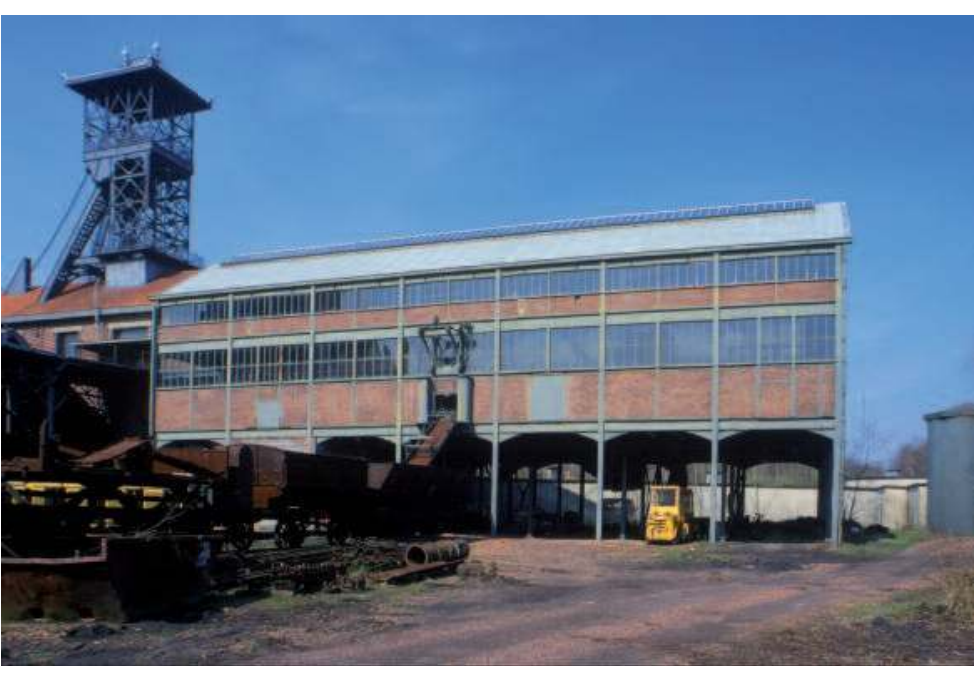

L'installation de triage-criblage du site minier de la fosse Delloye qui abrite le Centre historique minier de Lewarde. (c) Centre historique minier de Lewarde/Dahliette Sucheyre

* Agnès Mirambet-Paris est conservatrice du patrimoine au musée national de la Marine et de 1994 à 2006 au Centre historique minier de Lewarde a.paris@musee-marine.fr François Mirambet est ingénieur de recherche au Centre de recherche et de restauration des musées de France et au Laboratoire de recherche des Monuments historiques
Après avoir mis en évidence les difficultés de conservation-restauration spécifiques aux objets techniques et industriels, cette étude décrit - à travers l'exemple d'un projet de restauration de matériel ferroviaire - les grandes étapes d'un chantier de restauration puis, propose quelques solutions pour gérer au mieux ce type de dossier dans le respect de la loi sur les Musées de France.

En mars 2002, le Centre historique minier du NordPas-de-Calais à Lewarde proposait un colloque destiné à relancer à l'échelle nationale le débat sur la conservation-restauration du patrimoine technique et industriel et à remettre à jour l'état des lieux des pratiques en vigueur, en croisant les expériences des musées et des monuments historiques. Rappelons ici que les musées dits de société - musées techniques, musées de sites, écomusées, musées à collections historiques et militaires - ont cherché depuis trente ans à résoudre les nombreux problèmes rencontrés dans le cadre de la conservation-restauration des collections dont ils avaient la charge : matériels liés à toutes les activités industrielles possibles et imaginables (extraction minière, métallurgie, industrie textile, industrie du verre ou de la céramique, papeterie, agroalimentaire...), collections relatives aux transports (chemin de fer et autres transports en commun, véhicules automobiles, éléments relevant du domaine aéronautique, spatial ou maritime...). 
Sans aller jusqu’à présenter en détail les échanges survenus lors du colloque de Lewarde, qui ont fait l'objet d'une publication détaillée (1), nous rappellerons ici l'une des propositions faites lors de la table ronde de clôture. Il s'agissait d'analyser les différentes phases constitutives d'une opération de restauration et de lister les questions qui ne manquent pas de se poser à tout responsable d'objets relevant du patrimoine technique et industriel : à qui faut-il s'adresser ? Peut-on mener un travail de ce type en respectant la loi sur les Musées de France?

Nous souhaitons présenter ici un bilan des différentes expériences dont l'idée a pu germer lors de ce colloque et qui ont fait depuis l'objet de débats et d'échanges d'idées. Certes, il s'est avéré impossible que les responsables de musées volontaires au départ, souvent faute de temps ou de par les aléas liés à des mutations professionnelles, mènent un véritable chantier-pilote d'un bout à l'autre de la chaîne opératoire, depuis les recherches préalables sur l'objet jusqu'à la restauration en passant par la rédaction du cahier des charges, l'appel d'offres, la présentation du dossier en commission des Musées de France... Néanmoins les travaux engagés dans plusieurs musées ont permis d'obtenir des éléments de réponse concordants concernant les différentes phases de la méthodologie applicable au patrimoine technique et industriel, et cela dans le cadre de la loi sur les Musées de France. Dans le cadre de cet article, nous illustrerons les réflexions engagées à travers l'exemple d'un projet de restauration de matériel ferroviaire monté au Centre historique minier.

\section{Les problèmes de conservation inhérents au patrimoine technique et industriel}

Il est évident que s'efforcer de conserver sur le long terme des éléments du patrimoine est une entreprise complexe. En ce qui concerne le patrimoine technique et industriel, certaines de ses caractéristiques contribuent à accroître cette complexité. Rappelons en quoi les plus importantes d'entre elles consistent.

\section{Les problèmes liés à l'histoire} et à l'utilisation de l'objet

Une des principales difficultés rencontrées par les professionnels en charge de la conservation des éléments de ce patrimoine vient du fait que la plupart d'entre eux ont été conçus à l'origine pour fonctionner sur des périodes limitées avec des phases d'entretien et de maintenance répétées. Tout objet technique a connu une existence fondée sur la mise en fonctionnement et jalonnée par les différentes phases de son utilisation : sa mise en route, son entretien, les réparations, les ajustements, les modifications dont il a pu faire l'objet, puis son arrêt. À ce moment, qui a précédé son entrée au musée ou sa protection au titre des Monuments historiques, il a été privé du geste de l'ouvrier qui l'actionnait et l'entretenait. De nombreux matériaux d'origine ont été remplacés et certains matériaux employés n'ont pas été choisis pour résister à l'usure du temps au-delà de quelques décennies. À lissue de leur phase d'exploitation, ces matériels n'ont plus fait l'objet d'un entretien et cela a contribué à accélérer leur dégradation. Aussi de nombreux éléments sont-ils déjà fortement dégradés quand on décide de les « patrimonialiser ».

\section{Les difficultés liées à un environnement mal ou non contrôlé}

Très souvent, les éléments du patrimoine technique et industriel sont conservés dans des sites patrimoniaux, soit dans des bâtiments industriels, soit en extérieur. Dans le cas des métaux, la corrosion étant un phénomène thermodynamiquement privilégié, on constate que, sur ces sites, les vitesses de corrosion peuvent être élevées. De ce fait, conserver in situ ce type de patrimoine dans les conditions de conservation préventive préconisées dans les musées traditionnels se révèle très difficile à mettre en œuvre.

\section{L'association du métal}

avec des matériaux différents

De nombreux matériaux entrent dans la constitution des éléments de ce patrimoine. Ainsi, les métaux sont le plus souvent associés à des composants comme le bois, le cuir, les textiles, les matériaux polymères, la

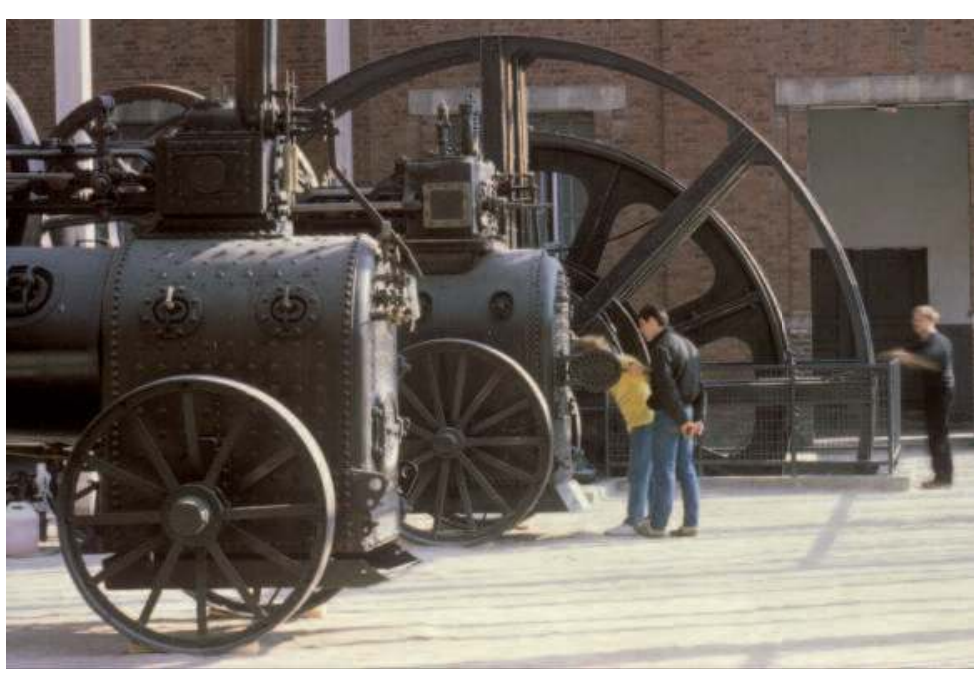

Comme de nombreux musées techniques, le Centre historique minier abrite différentes machines de grande taille placées en extérieur ou dans un environnement climatique difficilement contrôlable. (c) Centre historique minier de Lewarde/Dahliette Sucheyre 
pierre, les mortiers et les bétons. Or les normes de conservation et les traitements adaptés pour l'un des matériaux peuvent s'avérer nocifs pour d'autres qui lui sont associés. Par ailleurs, les matériaux utilisés pour certains objets ont été développés en vue de durées de service limitées et ils ne présentent pas une grande stabilité vis-à-vis de l'environnement. Cela rend la conservation de ces artefacts, dès lors qu'ils sont inscrits à l'inventaire des collections, difficile sur le long terme.

Les problèmes d'échelle du patrimoine industriel Compte tenu du fait que les éléments à conserver sont généralement de grandes dimensions ou en grand nombre, tout projet nécessite la mise en place de chantiers de restauration importants, avec des infrastructures lourdes : échafaudages, moyens de levage, grues, palans...

À l'inverse de la restauration de la peinture de chevalet qui peut s'appuyer sur une histoire longue, ayant permis de développer des protocoles et des méthodologies adaptées, la restauration appliquée aux matériels du patrimoine technique et industriel peut être considérée comme une discipline récente. Les méthodologies employées en la matière ne font pas l'objet d'échanges entre les professionnels de la restauration, ce qui ne facilite pas le développement de stratégies et de protocoles cohérents sur l'ensemble du territoire français.

Aujourd'hui les professionnels en charge de ce patrimoine s'interrogent sur la manière de faire restaurer leurs collections soit avec des personnels habilités mais ne disposant pas de moyens lourds adaptés, soit avec des entreprises qualifiées techniquement mais non habilitées (voir la figure ci-dessous).
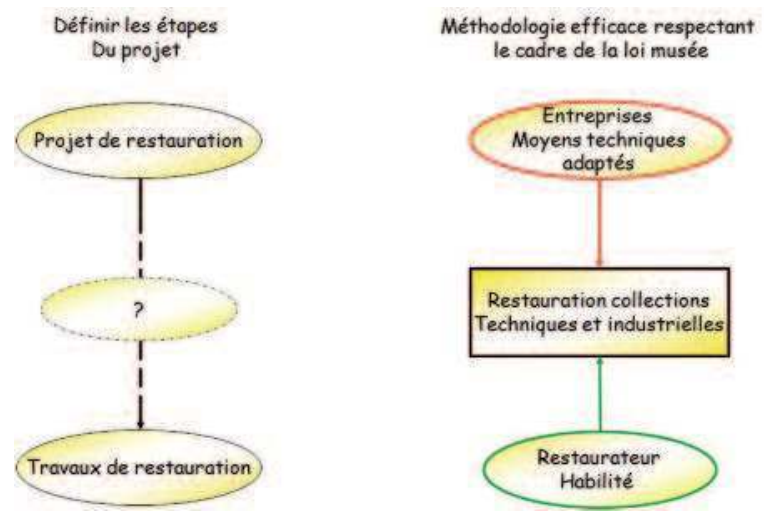

\section{De l'étude préalable à la présentation d'un devis devant la commission de restauration des Musées de France}

Le responsable de la conservation de l'objet doit d'abord s'interroger sur la manière dont l'élément à restaurer s'inscrit dans le projet scientifique et culturel de son établissement. Par exemple, la présentation de cet objet in situ peut imposer qu'il soit placé dans des conditions de conservation difficiles, lesquelles orienteront nécessairement le protocole de restauration. Autre exemple, le projet scientifique et culturel peut nécessiter la remise en fonctionnement d'une machine, un choix lourd de conséquences pour la mise en œuvre d'une restauration.

Pour illustrer notre propos, nous présenterons la démarche suivie lors d'un chantier monté au Centre historique minier de Lewarde sur des matériels ferroviaires. Les collections du musée comportent plusieurs locomotives et wagons provenant du patrimoine des Houillères du Bassin du Nord-Pas de Calais et présentant un degré de dégradation avancée. En 2002, lors du colloque évoqué précédemment, le Centre proposa d'ouvrir un chantier à ce sujet. Ces matériels devaient être à terme, après restauration, présentés in situ sur rails, soit en extérieur, dans la zone nord du site de la fosse Delloye, qui venait d'être requalifiée - le réseau ferré d'origine ayant été partiellement reconstitué - soit de manière plus abritée, du moins pour les pièces les plus " précieuses », sous l'atelier du triagecriblage. Rapidement trois wagons utilisés pour le transport du charbon ou des matières stériles, jugés particulièrement intéressants sur le plan historique en raison de leur rareté et présentant en outre un état de conservation très médiocre, furent sélectionnés pour faire l'objet du chantier.

Dans un premier temps, le projet scientifique et culturel étant ainsi arrêté, les différentes étapes du projet de restauration furent définies selon le schéma détaillé suivant.

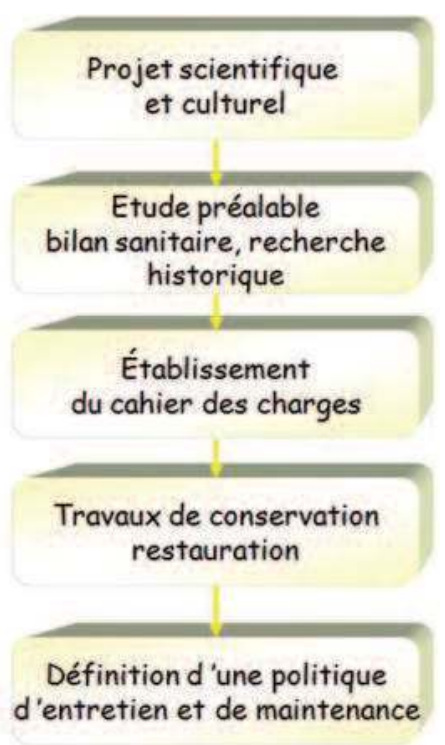




\section{Première étape, l'étude préalable et le bilan sanitaire}

Dès le début, il est apparu indispensable de mettre en place le principe d'une étude préalable aussi approfondie que possible, comprenant la réalisation d'une recherche historique et documentaire détaillée, un constat d'état intégrant un bilan sanitaire complet des matériels concernés pour permettre de définir le niveau de dégradation des matériaux ainsi qu'une couverture photographique. À cela s'ajouterait si possible un document concernant les interventions de réparation ou de restauration antérieures dans le but d'identifier pièces de remplacement et pièces originales.

Dans un premier temps, il fut envisagé de constituer un dossier documentaire sur chaque wagon, de manière à retracer son historique, à retrouver son constructeur et, dans la mesure du possible, à rassembler des plans susceptibles d'alimenter le cahier des charges de l'opération de restauration. L'étude documentaire devait s'appuyer sur une liste de sources relativement dispersées. Il s'agissait d'obtenir des renseignements sur les constructeurs - nous ignorions tout de deux d'entre eux - et de collecter autant que possible des données techniques et physiques (plans et procédés, marquages et couleurs) concernant les trois wagons, à intégrer au cahier des charges.

Une enquête fut alors menée dans les archives des compagnies minières et des HBNPC, conservés au Centre historique minier, mais également au Centre des Archives du monde du travail à Roubaix, aux Archives départementales du Nord (fonds de la compagnie d'Aniche), auprès de greffes de tribunaux, aux archives de la SNCF au Mans et à l'Institut national de la Propriété industrielle. Au total, la somme de documents retrouvée fut assez mince, dans la mesure où les archives des constructeurs avaient largement disparu. C'est encore le fonds du Centre historique minier qui livra le plus d'éléments intéressants, à savoir les plans et coupes de deux modèles de wagons proches des deux objets de l'étude. Malheureusement, aucun document relatif aux procédés de fabrication, aux marquages, aux peintures ne put être mis au jour. Concernant les recherches de couleur, quelques images repérées dans deux films en couleur tournés par les HBNPC fournirent des indications. Les souvenirs de plusieurs anciens employés des chemins de fer des Houillères furent également sondés, mais les résultats parurent peu fiables, car éminemment subjectifs. Du reste, il parut très vite évident que les Houillères ne réglementaient pas de manière stricte la couleur des matériels ferroviaires, et que, tout comme au fond de la mine, quand il fallait repeindre un wagon, on attrapait le premier bidon de peinture disponible sans se poser de questions, du gris généralement.

Cette situation se rencontre fréquemment dans le cadre du patrimoine industriel, ce qui implique de rester très prudent lors de la recherche d'éventuels résidus de peinture originelle.

Pour pallier le manque de plans relatifs au troisième wagon, un dessinateur industriel fut sollicité pour en établir. En corollaire de l'étude historique furent réalisés une couverture photographique et un bilan sanitaire dont on trouvera le détail phase par phase ci-dessous.

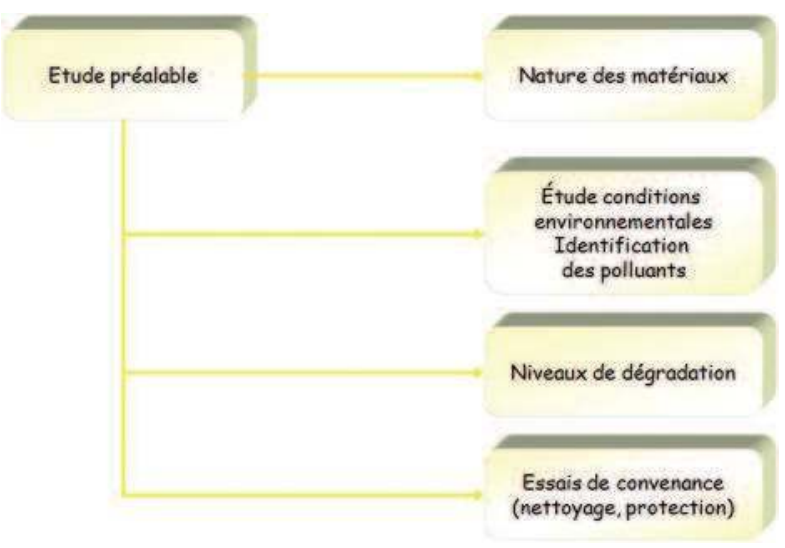

L'expérience menée au Centre historique minier et d'autres encore ont permis de dégager les éléments indispensables à tout bilan sanitaire concernant un objet technique. Dans un premier temps, une identification la plus complète possible des matériaux constitutifs est à entreprendre. Cette analyse permet de rechercher les moyens de conservation les plus adaptés. À titre d'exemple, il existe différentes compositions pour une même famille de matériaux métalliques ; ainsi certains éléments d'alliages peuvent-ils ralentir ou accélérer les cinétiques de corrosion. Il est donc nécessaire d’identifier avec précision le matériau pour définir de la manière la plus fiable possible les risques encourus.

D'autre part, la dégradation des matériaux étant très liée à la nature de l'environnement dans lequel l'objet est placé, une étude des conditions climatiques autour de la zone de conservation de l'objet doit être entreprise. Elle est indispensable à la fois au choix du protocole de restauration et à celui du protocole d'entretien et de maintenance. Des ré-applications plus ou moins 
rapprochées des systèmes de protection seront à mettre en place en fonction du niveau de corrosivité de l'environnement autour de la zone d'exposition.

Le niveau de dégradation des différents éléments doit être particulièrement bien quantifié afin de définir les zones qui seront à consolider - pour des problèmes de résistance mécanique - en particulier dans les cas d'une remise en mouvement ou bien pour des structures porteuses comme des chevalements. Mais l'étude permet également de rechercher des matériaux de substitution compatibles dans le cas où des consolidations à l'aide de pièces métalliques devront être réalisées.

Enfin des essais sur les méthodes de restauration envisagées (méthode de décapage, utilisation de nouveaux matériaux de consolidation ou de remplacement, applications de systèmes de protection) pourront être effectués pour déterminer le protocole de restauration prenant le mieux en compte différents facteurs : efficacité du traitement, aspect final, coûts. Ces essais sont indispensables dans le cas de grandes structures à traiter car ils permettent d'éviter des erreurs qui peuvent conduire à des dépenses importantes.

Pour les cas difficiles, notamment pour du patrimoine bâti, on préconisera le recrutement d'un maître d'œuvre chargé du suivi de l'ensemble de ces opérations.

\section{Deuxième étape, la rédaction du cahier des charges et le lancement de l'appel d'offres}

Les différents éléments recueillis permettent de rédiger un cahier des charges qui doit être le plus précis possible pour éviter toute dérive lors des travaux, en particulier de la part d'entreprises qui n'auraient pas d'expérience ou de qualification pour intervenir sur

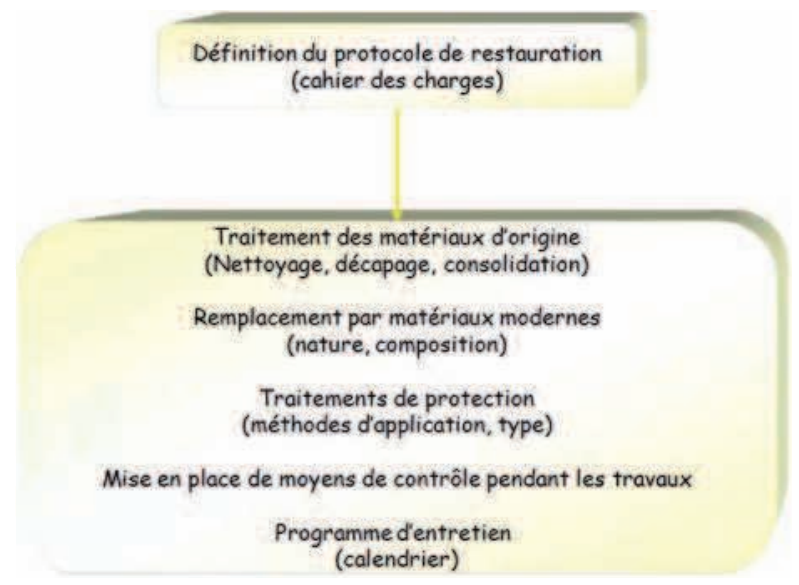

des objets patrimoniaux (voir le schéma ci-dessous). Insistons sur le fait que le cahier des charges doit tenir compte de tous les impératifs du projet scientifique et culturel, à savoir le lieu de stockage ou de mise en exposition envisagé, les conditions de présentation (mise en mouvement fréquente ou ponctuelle...). Pour des pièces de grandes dimensions, les risques vis-à-vis du public étant très importants, les problèmes de sécurité devront être également pris en compte.

Le document produit doit être le plus précis possible pour éviter toute erreur qui pourrait entraîner des surcoûts ou conduire à des mauvais choix lors de l'application d'un traitement de protection ou lors du remplacement de certains matériaux. Il présente le contexte général du projet, la nature de la proposition avec les données historiques et le bilan sanitaire puis la liste des interventions demandées et les protocoles à mettre en ouvre. Les conditions d'application des produits doivent également figurer sur le document afin de garantir la plus grande pérennité aux systèmes de protection choisis. Les entreprises doivent être capables à partir du document de parfaitement identifier les zones à risque et les problèmes spécifiques à prendre en compte pendant l'opération, la liste des types de produits souhaités ou à proscrire. Le cahier des charges peut servir en outre de check-list pour contrôler l'avancement des travaux et valider les différentes étapes de la restauration.

Après appel d'offres, le chantier de restauration proprement dit peut s'engager. Il faut alors organiser un suivi régulier afin de vérifier que les éléments du cahier des charges sont bien pris en compte. Pour des chantiers complexes portant sur des éléments de grande dimension ou des pièces en nombre important, on peut aussi prévoir, à certaines étapes-clés du chantier, des réunions avec un comité d'experts pour valider ou non les propositions.

Enfin, compte tenu de la spécificité du patrimoine industriel, il est impératif de mettre en place rapidement des procédures d'entretien et de maintenance pour éviter que les matériaux ne se dégradent à nouveau : ce point est particulièrement important dans le cas où les matériels restaurés sont destinés à être remis en fonctionnement. L'expérience montre que la maintenance est très souvent sous-estimée ou bien insuffisamment mise en œuvre faute de personnel formé pour cela.

Dans le cas des wagons du Centre historique minier, le cahier des charges regroupait classiquement l'étude 


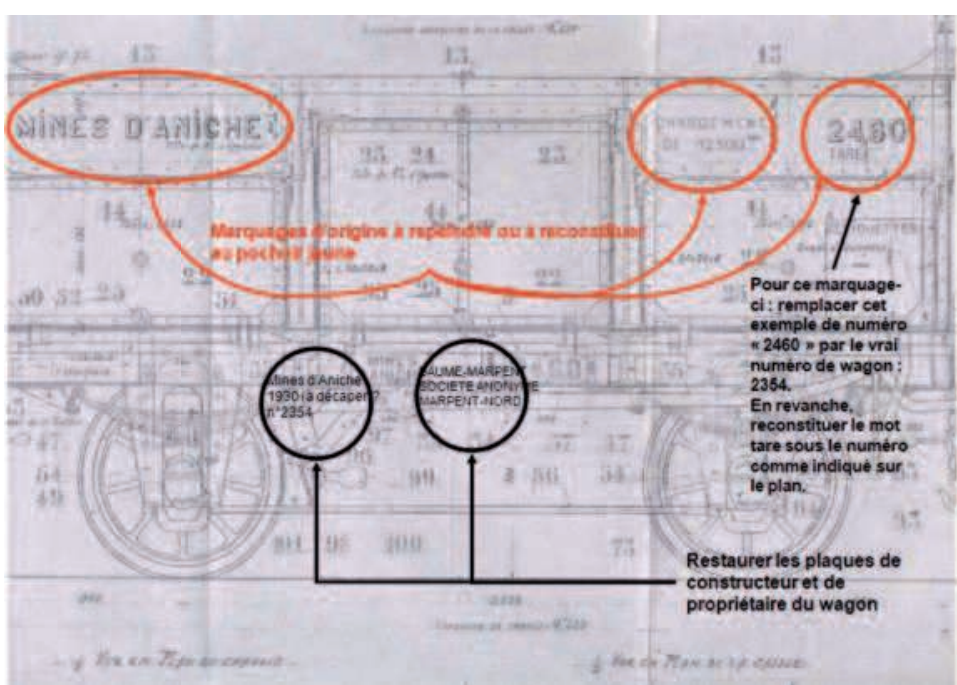

Les indications des travaux à faire sont portées sur le plan technique du wagon

(c) DR

historique, les plans des wagons (anciens et nouveaux), les photographies, le bilan sanitaire et des schémas indiquant le travail à faire sur les marquages de chaque wagon. Le souhait du conservateur, en l'occurrence, était de retrouver l'état des deux wagons provenant de la compagnie d'Aniche tels qu'ils se présentaient quand ils furent enregistrés dans le matériel roulant de cette compagnie et mis en service, durant l'Entredeux-guerres. Il faut dire que les plaques apposées par leurs constructeurs respectifs et par la compagnie d'Aniche qui les avait commandés, avaient été laissées en place au cours de leur deuxième vie dans le cadre des HBNPC, après la Seconde Guerre mondiale. Finalement, ces wagons n'avaient pas été beaucoup transformés au cours de leur longue carrière. Quant au wagon Miraillet, devant l'absence totale de plans anciens et de traces de marquages d'origine, on ne pouvait guère que restaurer les marquages des numéros d'enregistrement portés par la compagnie des mines de Lens - qui subsistaient sur le wagon lors de son arrivée au musée.

Le projet pour la présentation des wagons était le suivant : deux wagons devaient faire l'objet ponctuellement de démonstrations d'ouverture et de fermeture de leurs portes devant le public. Cette mise en mouvement devait être réalisée par des personnels des services techniques du musée formés à cet effet. Le troisième wagon semblait quant à lui trop fragile pour qu'il soit imaginé d'en mettre en mouvement certaines parties ; la présentation envisagée était donc statique à $100 \%$.
Il fut demandé aux entreprises sollicitées de fournir une proposition accompagnée de devis détaillés et, en complément, de fournir un chiffrage précis et clairement séparé pour chacun des trois wagons. En effet, selon l'importance du budget à engager, il était envisagé que les travaux soient décomposés en plusieurs tranches réparties sur plusieurs exercices budgétaires. Par ailleurs, il était demandé de fournir si possible plusieurs propositions de restitution en fonction de l'ampleur des altérations relevées. Le Centre historique minier joua le jeu en envoyant le cahier des charges, non seulement à des entreprises industrielles ayant déjà restauré du matériel ferroviaire, mais également à deux laboratoires de restauration des métaux faisant partie du réseau institutionnel, agréés « Musées de France ». L’un renvoya le cahier des charges faute de pouvoir répondre à la consultation, l'autre ne donna aucun signe de vie : leurs moyens techniques, adaptés au traitement d'éléments du patrimoine classique, ne leur permettaient pas de se lancer dans des travaux sur des
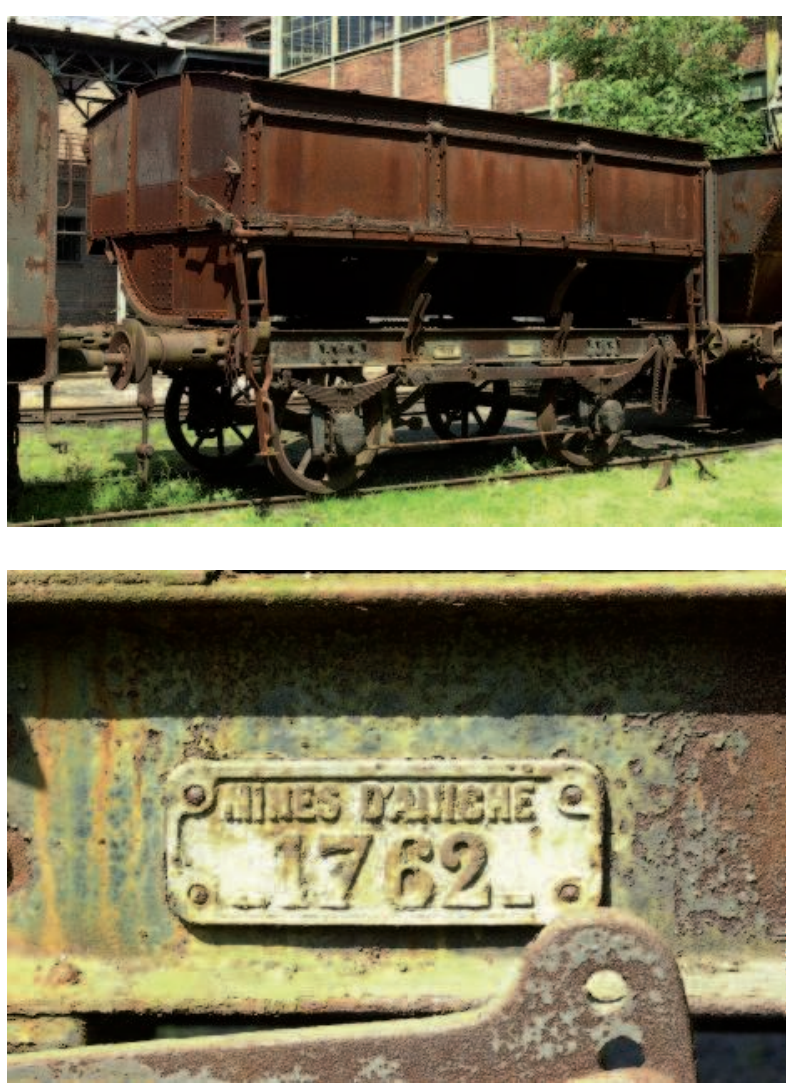

Le wagon dit "Panama » (type employé pour le creusement du canal de Panama, d'où son nom) utilisé pour la mise à terril, vue générale et vue de détail d'une plaque d'utilisateur à restaurer. (c) Centre historique minier de Lewarde/Dahliette Sucheyre

matériels aussi imposants. 
Dernière étape, la sélection

$d$ 'une entreprise et la présentation du dossier devant la commission de restauration des musées de France

Sans surprise, seules des entreprises industrielles répondirent à l'appel d'offres. La proposition jugée la plus en adéquation avec le cahier des charges émanait d'une entreprise de chaudronnerie industrielle qui avait déjà travaillé pour les Monuments historiques. Elle fut ensuite présentée devant la commission de restauration des Musées de France Picardie/Nord-Pas-de-Calais. L'entreprise pressentie n'ayant pas d'agrément pour intervenir sur des collections de Musées de France, la proposition incluait la mise sur pied d'un comité chargé d'analyser les différentes étapes du chantier de restauration. Ce comité avait été pensé de manière à intégrer les différents acteurs qui interviennent dans le domaine du patrimoine technique et industriel : experts représentant la Direction des Musées de France, les Monuments historiques ou la SNCF, praticiens de la restauration, personnes-ressources issues du monde associatif travaillant à l'étude ou la mise en valeur de matériels ferroviaires. Il faut souligner le fait que le dossier présenté en commission des Musées de France fut validé sans problèmes et qu'aucune discussion ne porta sur le fait que l'entreprise retenue, une chaudronnerie industrielle, bien qu'agréée par les Monuments historiques, ne faisait pas partie des listes de restaurateurs habilités à intervenir sur les collections des Musées de France. Il fut simplement demandé qu'un représentant du Centre de Recherche et de Restauration des Musées de France intègre le comité de suivi du chantier.

En 2006, le départ d'Agnès Paris, conservatrice ayant conduit le projet au Centre historique minier, a mis fin à l'expérimentation. Cependant, bien que la restauration même des objets n'ait pas eu lieu, la démarche entreprise a permis de mener à bon port la chaîne d'opérations prévue jusqu'à la présentation du cahier des charges et à la validation de l'offre d'une entreprise devant la commission des Musées de France.

\section{Restaurer le patrimoine industriel dans le cadre de la loi sur les Musées de France : une mission impossible?}

Comme on l'a vu plus haut, la restauration d'un objet relevant du patrimoine industriel est souvent subordonnée à la prise en compte d'un certain nombre de difficultés :

- la mise en place de protocoles adaptés au traitement de matériaux communément utilisés dans l'industrie au XIXe ou au XXe siècles (alliages complexes, matériaux composites) et de pièces de grandes dimensions ; - la mise en œuvre de techniques très spécifiques (soudure, réfection de pièces de structures) assortie de l'utilisation de moyens d'intervention lourds (levage, usinage, traitement de protection) ;

- l'intervention de professionnels à la fois formés et sensibilisés à la conservation de ce patrimoine et habilités dans le cadre de la loi sur les Musées de France.

Ces difficultés sont actuellement un frein au développement de projets de restauration autour du patrimoine industriel. Certes, il est tout à fait possible techniquement de faire appel à des structures possédant les compétences requises, citées précédemment. Ce sont généralement des entreprises qui interviennent lors de travaux de restauration pour les Monuments historiques. Cependant elles ne répondent pas aux critères posés par la loi sur les Musées de France, puisque leurs personnels n'ont pas été formés dans les institutions reconnues (MST, INP, école de Tours et d'Avignon...) et n'ont pas été sensibilisés aux règles déontologiques appliquées en conservation-restauration. À l'inverse, les personnels habilités n'ont pas, soit les compétences techniques pour travailler sur ce type de patrimoine, soit les moyens techniques nécessaires.

Cette situation est la conséquence des enseignements reçus dans les formations INP ou MST. En effet, la pratique de la restauration est plutôt axée sur des objets archéologiques ou des objets d'art de petite taille. Seuls certains ateliers du réseau qui

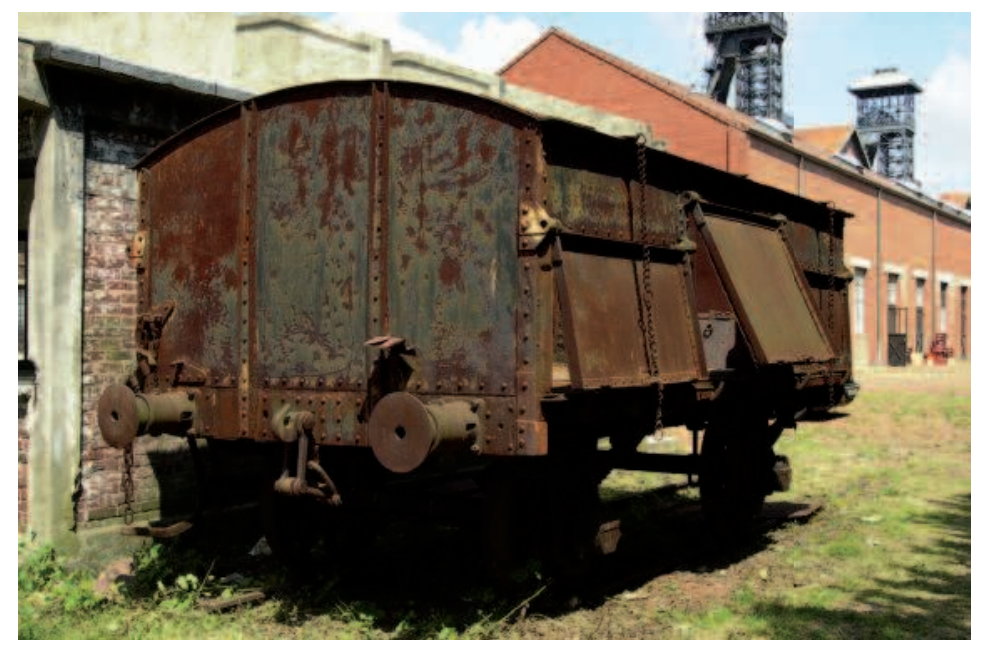

Le wagon de type Miraillet retenu pour l'étude. (c) Centre historique minier de Lewarde/Dahliette Sucheyre 
disposent de moyens un peu plus lourds ont travaillé sur des pièces plus importantes. De plus, la réalisation de tels travaux nécessite des moyens techniques très spécifiques, coûteux et difficiles à amortir pour des ateliers de restauration ou des restaurateurs indépendants et autres structures de ce type. C'est pourquoi, lors du montage de projets de restauration, les procédures d'appel d'offres lancées par les responsables de collections s'avèrent souvent infructueuses.

Il apparaît donc que, compte tenu du statut libéral des restaurateurs et des difficultés pour ceux-ci à disposer de moyens lourds car très onéreux, il est nécessaire de proposer des solutions alternatives.

Il est certain que les compétences techniques doivent plutôt être recherchées auprès des entreprises qui interviennent quotidiennement sur ces mêmes matériels et qui de ce fait disposent de moyens adaptés. Cependant, il est nécessaire d'introduire à leur pratique quotidienne une dose de déontologie pour respecter les principes qui fixent les limites et les règles en matière d'intervention. Il est certain que les personnels les plus qualifiés sont les restaurateurs issus des formations reconnues par la loi sur les Musées de France qui, au cours de leur cursus, ont été instruits sur les questions qui doivent être posées préalablement à toute intervention.

Dans un chantier type, des professionnels habilités seraient ainsi chargés de jouer un rôle d'expert auprès du responsable de collections pour le suivi des travaux ou la rédaction du cahier des charges. Pour que ce travail soit efficace, il est important d'engager une réflexion au sein des instituts de formation afin de sensibiliser les professionnels au cours du cursus mais également à travers des formations continues aux spécificités de la restauration de ce patrimoine. Des interactions plus fortes avec les corps de métiers qui travaillent sur des matériels de ce type (métallerie, chaudronnerie, mécanique) devront également être développées à l'avenir, dans la perspective d'acquérir les compétences techniques nécessaires.

Ce positionnement intermédiaire entre les pratiques des Monuments historiques et celles des musées apparaît comme la solution la plus aisée à mettre en œuvre. Aujourd'hui, le statut des restaurateurs les empêche de se lancer dans des chantiers lourds. Il faut donc rechercher une sorte de compromis entre capacité à traiter ces opérations de restauration et respecter à $100 \%$ les règles d'engagement des professionnels. À partir de ce constat, il convient de préciser comment l'interaction entre le restaurateur et l'entreprise pourrait se mettre en place au cours d'un chantier.
Nous proposons ici différentes solutions qui permettraient de travailler sur ce patrimoine tout en respectant les règles déontologiques appliquées en conservation-restauration :

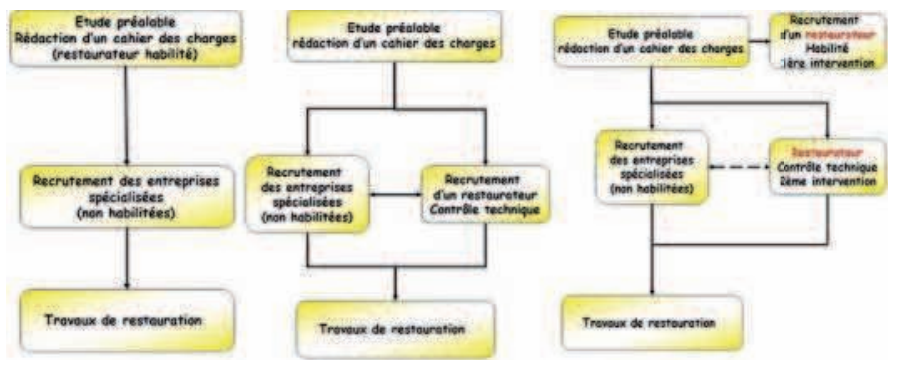

- première solution : travail d'un restaurateur en amont dans le cadre d'une étude préalable pouvant aboutir à la rédaction du cahier des charges préalable à la restauration. Puis dans un deuxième temps recrutement d'entreprises spécialisées non habilitées dans le cadre de la loi Musées de France ;

- deuxième solution : travail d'un restaurateur en amont dans le cadre d'une étude préalable pouvant aboutir à la rédaction d'un cahier des charges à la restauration; recrutement d'entreprises spécialisées non habilitées dans le cadre de la loi Musées de France avec suivi des travaux par un restaurateur qui contrôle la mise en place des travaux et réoriente en fonction des opérations ;

- troisième solution : recrutement dès le départ d'un consortium regroupant des restaurateurs et des entreprises spécialisées dans les champs non couverts par les spécialités des restaurateurs.

En parallèle, quelle que soit la solution retenue, un comité de suivi devrait être constitué pour aider à la mise en place des travaux en apportant des compétences sur des domaines variés - histoire des techniques, mécanique, physico-chimie, architecture pouvant orienter les choix en matière de conservation-restauration.
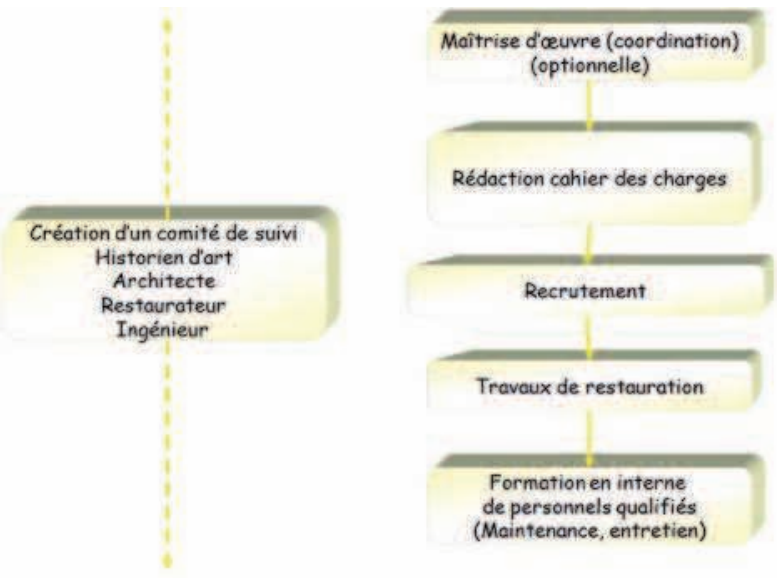
Il faut tout de même souligner que la mise en œuvre d'une procédure de ce type risque d'entraîner un surcoût à prendre en compte dans le montage d'un projet (frais de mission, de recherche...), ce qui risque d'être difficile à faire accepter par une collectivité locale ou un conseil d'administration.

\section{Conclusion}

On voit, à travers cette expérience, que les solutions proposées impliquent le partage de savoir-faire entre des professionnels formés au départ pour intervenir dans des champs différents. C'est encore plus vrai que dans d'autres domaines de la conservation-restauration car les expériences sur ce champ très particulier sont peu nombreuses : ce qui tend à dissuader les professionnels à s'engager dans le montage de telles opérations ${ }^{(2)}$.

Les différents problèmes auxquels nous avons été confrontés dans le cadre de ce travail de recherche autour de la conservation-restauration du patrimoine technique et industriel démontrent que c'est bien par le partage de compétences techniques acquises dans le domaine industriel et celles obtenues dans les écoles de formation à la restauration que pourront se développer pleinement des travaux de restauration.

Les auteurs tiennent à remercier l'ensemble des personnes avec lesquelles ils ont eu de fructueux échanges depuis l'organisation du colloque de Lewarde en 2002, tout spécialement Claudine Cartier, conservatrice générale à la direction générale des patrimoines, ainsi que : Jean-Louis Boutaine, France Dijoud, Michel Menu et Jean-Pierre Mohen au centre de recherche et de restauration des musées de France, Isabelle PallotFrossard, directrice du laboratoire de recherche des Monuments historiques, Christian Tilatti, conservateur au musée de l'Air et de l'Espace, ainsi que les équipes du musée des Salines de Salins-les Bains, de la Cité de la Dentelle de Calais, du musée de l'Histoire du Fer et du laboratoire d'archéologie des métaux de Nancy-Jarville. Ils expriment également leur gratitude à André Dubuc, directeur général du Centre historique minier de Lewarde, et à Virginie Debrabant, directrice du centre de ressources documentaires.

\section{Notes}

(1) La conservation du patrimoine technique et industriel. Actes du colloque du Centre historique minier de Lewarde. Lewarde : Éditions du Centre historique minier, 2002, 160 p.

(2) Pour tout renseignement concernant des projets de conservation-restauration du patrimoine technique et industriel, contacter Philippe Goergen, conservateur du patrimoine au C2RMF : philippe.goergen@culture.gouv.fr.

\section{Bibliographie}

Dubus, M., Mirambet, F. et Paris, A. La préservation du patrimoine technique et industriel : entre conservation préventive et protocoles d'entretien, Technè, n¹9, 2004, pp. 123-129.

La conservation du patrimoine technique et industriel, actes du colloque du Centre historique minier de Lewarde, éditions du Centre historique minier avec le soutien de la mission de la recherche et de la technologie du ministère de la Culture, décembre 2002, 160 p.

Leluc, S., Mirambet, F., Paris, A. et Texier, A Conservation-restauration des éléments métalliques du patrimoine technique et industriel. La mise en place de chantiers-pilotes, Technè, nº18, 2003, pp. 101-105.

Rolland-Villemot, B. Le traitement des collections industrielles et techniques, de la connaissance à la diffusion, la Lettre de l'OCIM, $\mathrm{n}^{\circ} 73$, janvier-février 2001, pp. 13-18.

Vincent, S. La restauration et la conservation des machines industrielles : analyse des pratiques à partir de l'exemple de quelques musées français, Musées et sociétés, actes du colloque de Mulhouse-Ungersheim, Direction des Musées de France, 1993, pp. 125-128. 